\title{
Sex Differences in the Outcomes of Elderly Patients with Acute Coronary Syndrome
}

\author{
Shi Tai, ${ }^{1}$ Xuping Li, ${ }^{1}$ Hui Yang, ${ }^{1}$ Zhaowei Zhu, ${ }^{1}$ Liang Tang, ${ }^{1}$ Liyao Fu, ${ }^{2}$ Xinqun Hu $\mathbb{D},{ }^{1}$ \\ Zhenfei Fang $\mathbb{D}^{1}{ }^{1}$ Yonghong Guo $\mathbb{D}^{3},^{3}$ and Shenghua Zhou $\mathbb{D}^{1}$ \\ ${ }^{1}$ Department of Cardiology, The Second Xiangya Hospital of Central South University, Changsha, China \\ ${ }^{2}$ Department of Blood Transfusion, The Second Xiangya Hospital of Central South University, Changsha, China \\ ${ }^{3}$ Department of Geriatrics, The Second Xiangya Hospital of Central South University, Changsha, China
}

Correspondence should be addressed to Yonghong Guo; guoyhong@sina.cn and Shenghua Zhou; zhoushenghua@csu.edu.cn

Received 29 November 2019; Revised 25 March 2020; Accepted 6 April 2020; Published 12 May 2020

Guest Editor: Zhen Yang

Copyright (@ 2020 Shi Tai et al. This is an open access article distributed under the Creative Commons Attribution License, which permits unrestricted use, distribution, and reproduction in any medium, provided the original work is properly cited.

Background. The impact of sex on the outcome of patients with acute coronary syndrome (ACS) has been suggested, but little is known about its impact on elderly patients with ACS. Methods. This study analyzed the impact of sex on in-hospital and 1-year outcomes of elderly ( $\geq 75$ years of age) patients with ACS hospitalized in our department between January 2013 and December 2017. Results. A total of 711 patients were included: 273 (38.4\%) women and 438 (61.6\%) men. Their age ranged from 75 to 94 years, similar between women and men. Women had more comorbidities (hypertension $(79.5 \%$ vs. $72.8 \%, p=0.050)$, diabetes mellitus (35.2\% vs. $26.5 \%, p=0.014)$, and hyperuricemia $(39.9 \%$ vs. $32.4 \%, p=0.042)$ ) and had a higher prevalence of non-STsegment elevation ACS (NSTE-ACS) $(79.5 \%$ vs. $71.2 \%, p=0.014)$ than men. The prevalence of current smoking $(56.5 \%$ vs. $5.4 \%$, $p<0.001)$, creatinine levels ( $124.4 \pm 98.6$ vs. $89.9 \pm 54.1, p<0.001)$, and revascularization rate $(39.7 \%$ vs. $30.0 \%, p=0.022)$ were higher, and troponin TnT and NT-proBNP tended to be higher in men than in women. The in-hospital mortality rate was similar ( $3.5 \%$ vs. $4.4 \%, p=0.693)$, but the 1 -year mortality rate was lower in women than in men $(14.7 \%$ vs. $21.7 \%, p=0.020)$. The multivariable analysis showed that female sex was a protective factor for 1 -year mortality in all patients $(\mathrm{OR}=0.565,95 \% \mathrm{CI}$ $0.351-0.908, p=0.018)$ and in patients with STEMI $(\mathrm{OR}=0.416,95 \%$ CI $0.184-0.940, p=0.035)$ after adjustment. Conclusions. Among the elderly patients with ACS, the 1-year mortality rate was lower in women than in men, which could be associated with comorbidities and ACS type.

\section{Introduction}

Cardiovascular disease (CVD) is the leading cause of death for both men and women worldwide [1]. Acute coronary syndrome (ACS), a major clinical manifestation of atherothrombosis, refers to a wide spectrum of clinical presentations, such as ST-segment elevation myocardial infarction (STEMI) and non-ST-segment elevation ACS (NSTE-ACS), and increases with age, and the outcomes of ACS in elderly patients are generally worse than those in young patients [2]. Almost one out of every two patients hospitalized for ACS is over 75 years of age $[3,4]$, and the inhospital mortality due to ACS ranges from $4 \%$ to10\% $[4,5]$.

Women account for approximately $30 \%$ of patients presenting with ACS $[4,6]$ and have long been described as being "older and sicker" than their male counterparts [7]. In particular, significant sex-related differences exist in ACS presentation, management, and outcomes [7]. A large contemporary registry study in China demonstrated that women hospitalized for ACS received acute treatments and secondary prevention less frequently and had a higher inhospital mortality than men due to poor clinical profiles and little evidence for acute treatments [4]. To date, little is known about the sex-related differences in elderly patients with ACS.

The Italian Elderly ACS study included patients with NSTE-ACS and $\geq 75$ years of age and showed that women had poor 1-year primary outcomes, including death, nonfatal myocardial infarction, disabling stroke, cardiac 
rehospitalization, and severe bleeding [8]. According to a nationwide registry study in the Netherlands, the relation between sex and mortality appeared to be age-dependent, with increased mortality in women at a young age and decreased mortality in women at an advanced age [9]. It is still unclear whether these differences can be solely explained by sex or by other factors such as age, extent or impact of risk factors, clinical presentation, and treatment strategies. Therefore, this study focused on patients with ACS and $\geq 75$ years and aimed to investigate the sex differences in the clinical characteristics, in-hospital management, adverse events, and 1-year mortality among those patients.

\section{Methods}

2.1. Study Design and Patients. This retrospective singlecenter study included 711 consecutive patients with ACS and $\geq 75$ years of age who were hospitalized in our department between January 2013 and December 2017. Only patients who were initially admitted to our center were included; those who were transferred from other centers were excluded. The diagnostic criteria for ACS were based on the presence of chest pain or discomfort, electrocardiogram (ECG) findings, and myocardial injury biomarker measurements. The study protocol conformed to the ethical guidelines of the 1975 Declaration of Helsinki and was approved by the Human Research Committee of the Second Xiangya Hospital of Central South University. The need for individual consent was waived by the committee.

2.2. Definitions and Endpoints. ACS was defined in accordance with the guidelines published by the American College of Cardiology for the diagnosis and management of patients with ST-segment elevation myocardial infarction (STEMI) and non-ST-segment elevation ACS (NSTE-ACS) $[10,11]$. Renal insufficiency $(\mathrm{CKD} \geq 3)$ was defined as an estimated glomerular filtration rate $(e G F R)<60 \mathrm{~mL} / \mathrm{min}$ per $1.73 \mathrm{~m}^{2}$. In our study, severe heart failure indicated class IIIIV heart failure, according to the Killip or New York Heart Association classification system. Readmission was defined as any readmission after discharge. Stroke was defined as the sudden onset of focal neurological deficit resulting from either cerebral infarction or hemorrhage. According to the Bleeding Academic Research Consortium (BARC) criteria, BARC types 2 and 3 were included as in-hospital bleeding events [12]. A BARC type 2 event was defined as clinically overt hemorrhage requiring medical attention, whereas a BARC type 3 event was defined as bleeding, including gastrointestinal bleeding, respiratory bleeding, and genitourinary bleeding, with a hemoglobin decrease of at least $3 \mathrm{~g} /$ $\mathrm{dl}$, requiring transfusion or surgical intervention.

The primary outcome was 1-year all-cause mortality. The secondary outcomes included the rates of revascularization, readmission, and stroke over 1 year of follow-up.

2.3. Data Collection. The following data were collected: body weight, height, and body mass index (BMI) during hospitalization, diabetes, atrial fibrillation, chronic kidney disease, history of chest pain, history of CVD treatment, smoking, laboratory parameters, length of hospital stay, demographic characteristics, medication, in-hospital management, and adverse events. The following biochemical parameters were also extracted from the medical charts: hemoglobin, total cholesterol, high-density lipoprotein cholesterol (HDL-C), low-density lipoprotein cholesterol (LDL-C), triglycerides, and glycated hemoglobin (HbA1c).

2.4. Follow-Up. In-hospital outcomes were ascertained by a hospital chart review. After discharge, participant follow-up was carried out by means of outpatient visits and telephone calls for up to one year.

2.5. Statistical Analysis. Categorical variables were presented as numbers (percentages) and compared using the Pearson chi-squared tests or Fisher's exact test. Continuous variables were presented as mean \pm SD and compared using Student's $t$-test if the data were normally distributed. Nonnormally distributed continuous variables were presented as medians (interquartile ranges) and compared using nonparametric tests. Odds ratios (ORs) were presented with 95\% confidence intervals (CIs). Univariable factor logistic regression was used to analyze the risk factors associated with 1-year allcause mortality. A multivariable logistic regression analysis was used to define the independent determinants of 1-year all-cause mortality after adjusting for comorbidities, presentation, and clinical profiles. Meaningful factors, defined by the univariable $p<0.05$, including age (Model 1); hypertension; current smoking; severe heart failure (Model 2); hemoglobin, platelet, total cholesterol, HDL-C, LDL-C, creatinine, and serum uric acid levels (Model 3), were included in the multivariable logistic regression analysis. A two-tailed $p$-value $<0.05$ indicated statistical significance. All statistical analyses were conducted using SPSS 22.0 (IBM, Armonk, NY, USA).

\section{Results}

3.1. Characteristics of the Patients. There were 273 (38.4\%) women and $438(61.6 \%)$ men. They were $75-94$ years of age, and the age distribution was similar between women and men (78 [76-81] vs. 78 [76-80], $p=0.381)$.

3.2. Characteristics of the Patients according to Sex. The baseline characteristics of each group are listed in Table 1. Women had a higher prevalence of traditional risk factors for CVD, including hypertension $(79.5 \%$ vs. $72.8 \%$, $p=0.050)$, diabetes mellitus $(35.2 \%$ vs. $26.5 \%, p=0.014)$, and hyperuricemia (39.9\% vs. $32.4 \%, p=0.042)$, but a lower prevalence of current smoking $(5.4 \%$ vs. $56.5 \%, p<0.001)$ than men. The prevalence of severe heart failure on admission (56.8\% vs. $46.0 \%, p=0.005)$ and NSTE-ACS $(79.5 \%$ vs. $71.2 \%, p=0.014)$ was significantly higher, while STEMI (20.5\% vs. $28.8 \%, p=0.014)$ was less frequent in women than in men. Platelet counts $(202.9 \pm 64.9$ vs. $174.3 \pm 61.4$, $p<0.001)$, total cholesterol ( $4.1 \pm 1.0$ vs. $3.8 \pm 0.9, p<0.001)$, 
TABle 1: Differences between women and men.

\begin{tabular}{|c|c|c|c|}
\hline & Women $(n=273)$ & Men $(n=438)$ & $p$ \\
\hline \multicolumn{4}{|l|}{ Demographics and medical history } \\
\hline Age, yrs (median, IQR) & $78(76-81)$ & $78(76-80)$ & 0.381 \\
\hline Body mass index (median, IQR) & $22.7(20.6-25.4)$ & $23.1(20.9-25.4)$ & 0.427 \\
\hline Diabetes mellitus, no. (\%) & $96(35.2)$ & $116(26.5)$ & 0.014 \\
\hline Hypertension, no. (\%) & $217(79.5)$ & $319(72.8)$ & 0.050 \\
\hline Atrial fibrillation, no. (\%) & $30(11.2)$ & $53(12.3)$ & 0.656 \\
\hline $\mathrm{CKD} \geq 3$ & $13(4.8)$ & $26(5.9)$ & 0.506 \\
\hline Stroke, no. (\%) & $32(11.9)$ & $71(16.5)$ & 0.098 \\
\hline Previous chest pain, no. (\%) & $181(67.5)$ & $286(66.2)$ & 0.716 \\
\hline Previous PCI, no. (\%) & $36(13.5)$ & $78(18.1)$ & 0.112 \\
\hline Previous CABG, no. (\%) & $3(1.1)$ & $10(2.3)$ & 0.255 \\
\hline Current smoking, no. (\%) & $14(5.4)$ & $239(56.5)$ & $<0.001$ \\
\hline \multicolumn{4}{|l|}{ Clinical presentation } \\
\hline Hyperuricemia, no. (\%) & $109(39.9)$ & $142(32.4)$ & 0.042 \\
\hline Severe heart failure, no. (\%) & $155(56.8)$ & $201(46.0)$ & 0.005 \\
\hline STEMI, no. (\%) & $56(20.5)$ & $126(28.8)$ & 0.014 \\
\hline NSTE-ACS, no. (\%) & $217(79.5)$ & $312(71.2)$ & 0.014 \\
\hline \multicolumn{4}{|l|}{ Laboratory data } \\
\hline WBC, $10^{9} / \mathrm{L}($ mean $\pm \mathrm{SD})$ & $7.4 \pm 3.3$ & $7.2 \pm 3.2$ & 0.568 \\
\hline Hemoglobin, g/L (mean $\pm \mathrm{SD})$ & $110.4 \pm 18.3$ & $121.8 \pm 20.2$ & $<0.001$ \\
\hline Platelets, $10^{9} / \mathrm{L}($ mean $\pm \mathrm{SD})$ & $202.9 \pm 64.9$ & $174.3 \pm 61.4$ & $<0.001$ \\
\hline Fasting glucose, mmol/l (median, IQR) & $6.0(4.9-7.6)$ & $5.7(4.8-7.1)$ & 0.181 \\
\hline HbA1C, \% (median, IQR) & $6.7(6.0-7.4)$ & $6.1(5.6-6.5)$ & 0.005 \\
\hline ALT, u/l (median, IQR) & $17.5(11.7-28.3)$ & $19.9(13.6-33.5)$ & 0.012 \\
\hline AST, u/l (median, IQR) & $22.6(17.1-39.5)$ & $22.6(17.8-49.9)$ & 0.579 \\
\hline Albumin, g/L (mean $\pm S D)$ & $35.1 \pm 4.5$ & $35.1 \pm 4.1$ & 0.902 \\
\hline Triglycerides, $\mathrm{mmol} / \mathrm{l}($ mean $\pm \mathrm{SD})$ & $1.6 \pm 1.0$ & $1.6 \pm 4.2$ & 0.960 \\
\hline Total cholesterol, mmol/l $($ mean $\pm \mathrm{SD})$ & $4.1 \pm 1.0$ & $3.8 \pm 0.9$ & $<0.001$ \\
\hline $\mathrm{HDL}-\mathrm{C}, \mathrm{mmol} / \mathrm{l}($ mean $\pm \mathrm{SD})$ & $1.1 \pm 0.3$ & $1.0 \pm 0.3$ & 0.002 \\
\hline $\mathrm{LDL}-\mathrm{C}, \mathrm{mmol} / \mathrm{l}(\mathrm{mean} \pm \mathrm{SD})$ & $2.4 \pm 0.9$ & $2.2 \pm 0.8$ & 0.002 \\
\hline Creatinine, $\mu \mathrm{mmol} / \mathrm{l}($ mean $\pm \mathrm{SD})$ & $89.9 \pm 54.1$ & $124.4 \pm 98.6$ & $<0.001$ \\
\hline Serum uric acid, $\mu \mathrm{mmol} / \mathrm{l}($ mean $\pm \mathrm{SD})$ & $349.2 \pm 119.1$ & $381.4 \pm 110.1$ & $<0.001$ \\
\hline $\mathrm{PT}$, sec $($ mean $\pm \mathrm{SD})$ & $13.7 \pm 4.3$ & $13.5 \pm 2.8$ & 0.674 \\
\hline $\mathrm{APTT}, \sec ($ mean $\pm \mathrm{SD})$ & $39.5 \pm 14.4$ & $41.4 \pm 22.2$ & 0.562 \\
\hline $\mathrm{CK}-\mathrm{Mb}, \mathrm{u} / \mathrm{l}(\mathrm{mean} \pm \mathrm{SD})$ & $35.0 \pm 71.4$ & $44.3 \pm 119.0$ & 0.266 \\
\hline TnT, pg/ml (median, IQR) & $19.3(9.7-603.3)$ & $33.2(12.3-1461.0)$ & 0.141 \\
\hline hs-CRP, mg/l (median, IQR) & $4.3(1.3-23.2)$ & $5.1(1.4-20.2)$ & 0.849 \\
\hline NT-proBNP, pg/ml (median, IQR) & $935.9(290.4-3082.5)$ & $1337.0(394.9-3737.7)$ & 0.067 \\
\hline $\mathrm{EF}, \%($ mean $\pm \mathrm{SD})$ & $53 \pm 10.4$ & $53 \pm 10.9$ & 0.460 \\
\hline \multicolumn{4}{|l|}{ In-hospital management } \\
\hline Aspirin, no. (\%) & $227(83.2)$ & $376(86.2)$ & 0.262 \\
\hline Clopidogrel, no. (\%) & $234(86.0)$ & $391(89.5)$ & 0.168 \\
\hline ACEI/ARB, no. (\%) & $168(61.5)$ & $268(61.2)$ & 0.925 \\
\hline Beta blocker, no. (\%) & $206(75.5)$ & $306(69.9)$ & 0.106 \\
\hline Statin, no. (\%) & $265(97.8)$ & $422(96.3)$ & 0.283 \\
\hline Diuretic, no. (\%) & $128(47.6)$ & $210(48.6)$ & 0.791 \\
\hline PPI, no. (\%) & $227(84.4)$ & $340(78.7)$ & 0.063 \\
\hline IABP, no. (\%) & $7(2.6)$ & $18(4.2)$ & 0.255 \\
\hline Revascularization & $82(30.0)$ & $174(39.7)$ & 0.022 \\
\hline \multicolumn{4}{|l|}{ In-hospital events } \\
\hline Heart failure, no. (\%) & $42(15.7)$ & $77(17.9)$ & 0.458 \\
\hline Bleeding, no. (\%) & $22(8.2)$ & $39(9.1)$ & 0.706 \\
\hline Ventricular tachycardia, no. (\%) & $23(8.6)$ & $36(8.3)$ & 0.920 \\
\hline Stroke, no. (\%) & $5(1.9)$ & $2(0.5)$ & 0.071 \\
\hline Death, no. (\%) & $4(3.5)$ & $8(4.4)$ & 0.693 \\
\hline \multicolumn{4}{|l|}{ One-year follow-up } \\
\hline Revascularization & $4(1.6)$ & $7(1.9)$ & 0.801 \\
\hline Readmission, no. (\%) & $96(36.9)$ & $159(39.9)$ & 0.436 \\
\hline Stroke, no. (\%) & $13(5.2)$ & $18(4.8)$ & 0.827 \\
\hline Death, no. (\%) & $40(14.7)$ & $95(21.7)$ & 0.020 \\
\hline
\end{tabular}

STEMI: ST-segment elevation myocardial infarction; NSTE-ACS: non-ST-elevation acute coronary syndrome; WBC: white blood cell; ALT: glutamic-pyruvic transaminase; AST: glutamic-oxaloacetic transaminase; HDL-C: high-density lipoprotein cholesterol; LDL-C: low-density lipoprotein cholesterol; PT: prothrombin time; APTT: activated partial thromboplastin time; ACEI/ARB: angiotensin-converting enzyme inhibitors/angiotensin receptor blockers; PPI: proton pump inhibitor; IABP: intra-aortic balloon pump; CKD: chronic kidney disease; and $\mathrm{CKD} \geq 3$ : estimated glomerular filtration rate (eGFR) $<60 \mathrm{~mL} / \mathrm{min}$ per $1.73 \mathrm{~m}$. 
TABLE 2: Differences between women and men according to ACS type.

\begin{tabular}{|c|c|c|c|c|c|c|}
\hline & \multicolumn{3}{|c|}{ NSTE-ACS } & \multicolumn{3}{|c|}{ STEMI } \\
\hline & Women $(n=217)$ & Men $(n=312)$ & $p$ & Women $(n=56)$ & Men $(n=126)$ & $p$ \\
\hline \multicolumn{7}{|l|}{$\begin{array}{l}\text { Demographics and medical } \\
\text { history }\end{array}$} \\
\hline Age, yrs (median, IQR) & $78(76-81)$ & $78(76-80)$ & 0.085 & $77(76-80)$ & $78(76-81)$ & 0.218 \\
\hline $\begin{array}{l}\text { Body mass index, (median, } \\
\text { IQR) }\end{array}$ & $22.7(20.5-25.4)$ & $23.2(20.9-25.5)$ & 0.275 & $22.3(20.7-25.3)$ & $22.3(19.8-24.6)$ & 0.703 \\
\hline Diabetes mellitus, no. (\%) & $69(31.8)$ & $91(29.2)$ & 0.517 & $27(48.2)$ & $25(19.8)$ & $<0.001$ \\
\hline Hypertension, no. (\%) & $182(83.7)$ & $248(79.5)$ & 0.204 & $35(62.5)$ & $71(56.3)$ & 0.437 \\
\hline Atrial fibrillation, no. (\%) & $19(8.9)$ & $35(11.4)$ & 0.362 & $11(19.6)$ & $18(14.4)$ & 0.374 \\
\hline $\mathrm{CKD} \geq 3$ & $9(4.2)$ & $14(4.5)$ & 0.853 & $4(7.1)$ & $12(9.5)$ & 0.601 \\
\hline Stroke, no. (\%) & $25(11.8)$ & $54(17.6)$ & 0.068 & $7(12.5)$ & $17(13.7)$ & 0.825 \\
\hline Previous chest pain, no. (\%) & $148(69.8)$ & $219(71.3)$ & 0.708 & $33(58.9)$ & $67(53.6)$ & 0.505 \\
\hline Previous PCI, no. (\%) & $32(15.1)$ & $69(22.5)$ & 0.037 & $4(7.3)$ & $9(7.2)$ & 0.986 \\
\hline Previous CABG, no. (\%) & $3(1.4)$ & $10(3.3)$ & 0.187 & $0(0)$ & $0(0)$ & NA \\
\hline Current smoking, no. (\%) & $7(3.4)$ & $166(55.5)$ & $<0.001$ & $7(12.5)$ & $73(58.9)$ & $<0.001$ \\
\hline \multicolumn{7}{|l|}{ Clinical presentation } \\
\hline Hyperuricemia, no. (\%) & $91(41.9)$ & $98(31.4)$ & 0.013 & $18(32.1)$ & $44(34.9)$ & 0.715 \\
\hline Severe heart failure, no. (\%) & $132(60.8)$ & $149(47.9)$ & 0.003 & $23(41.1)$ & $52(41.3)$ & 0.980 \\
\hline \multicolumn{7}{|l|}{ Laboratory data } \\
\hline $\mathrm{WBC}, 10^{9} / \mathrm{L}($ mean $\pm \mathrm{SD})$ & $6.8 \pm 2.6$ & $6.5 \pm 2.4$ & 0.076 & $9.4 \pm 4.6$ & $9.1 \pm 4.0$ & 0.752 \\
\hline Hemoglobin, $g / L($ mean $\pm S D)$ & $110.3 \pm 19.0$ & $122.4 \pm 20.2$ & $<0.001$ & $110.9 \pm 15.4$ & $120.3 \pm 20.1$ & 0.001 \\
\hline Platelets, $10^{9} / \mathrm{L}($ mean \pm SD $)$ & $200.3 \pm 62.3$ & $171.0 \pm 60.0$ & $<0.001$ & $212.7 \pm 73.7$ & $182.5 \pm 64.2$ & 0.009 \\
\hline $\begin{array}{l}\text { Fasting glucose, mmol/l } \\
\text { (median, IQR) }\end{array}$ & $5.9(4.9-6.9)$ & $5.6(4.8-6.8)$ & 0.083 & $6.8(4.9-8.2)$ & $6.3(5.3-7.7)$ & 0.873 \\
\hline HbA1C, \% (median, IQR) & $6.5(5.9-7.0)$ & $6.2(5.7-6.8)$ & 0.115 & $7.0(6.1-7.7)$ & $5.8(5.5-6.1)$ & 0.035 \\
\hline ALT, u/l (median, IQR) & $16.0(10.9-22.9)$ & $18.0(12.4-27.8)$ & 0.006 & $33.0(20.7-48.5)$ & $31.7(16.7-58.8)$ & 0.533 \\
\hline AST, $\mathrm{u} / \mathrm{l}$ (median, IQR) & $21.0(16.2-26.9)$ & $19.8(16.9-27.2)$ & 0.813 & $76.0(25.7-286.4)$ & $67.3(28.7-187.7)$ & 0.759 \\
\hline Albumin, g/L (mean $\pm S D)$ & $35.7 \pm 4.5$ & $35.8 \pm 3.8$ & 0.785 & $32.9 \pm 3.5$ & $33.4 \pm 4.1$ & 0.494 \\
\hline $\begin{array}{l}\text { Triglycerides, } \mathrm{mmol} / \mathrm{l} \\
(\text { mean } \pm \text { SD })\end{array}$ & $1.6 \pm 1.1$ & $1.7 \pm 5.0$ & 0.725 & $1.6 \pm 0.8$ & $1.3 \pm 0.9$ & 0.009 \\
\hline $\begin{array}{l}\text { Total cholesterol, } \mathrm{mmol} / \mathrm{l} \\
(\mathrm{mean} \pm \mathrm{SD})\end{array}$ & $4.0 \pm 1.0$ & $3.7 \pm 0.9$ & $<0.001$ & $4.4 \pm 1.1$ & $3.9 \pm 0.9$ & 0.002 \\
\hline $\begin{array}{l}\text { HDL-cholesterol, } \mathrm{mmol} / \mathrm{l} \\
(\mathrm{mean} \pm \mathrm{SD})\end{array}$ & $1.1 \pm 0.3$ & $1.0 \pm 0.3$ & 0.002 & $1.1 \pm 0.3$ & $1.0 \pm 0.3$ & 0.373 \\
\hline $\begin{array}{l}\text { LDL-cholesterol, } \mathrm{mmol} / \mathrm{l} \\
(\mathrm{mean} \pm \mathrm{SD})\end{array}$ & $2.3 \pm 0.9$ & $2.2 \pm 0.8$ & 0.018 & $2.7 \pm 1.0$ & $2.3 \pm 0.8$ & 0.005 \\
\hline $\begin{array}{l}\text { Creatinine, } \mu \mathrm{mmol} / \mathrm{l} \\
(\mathrm{mean} \pm \mathrm{SD})\end{array}$ & $91.3 \pm 53.7$ & $124.7 \pm 109.1$ & $<0.001$ & $84.3 \pm 56.0$ & $123.4 \pm 66.6$ & $<0.001$ \\
\hline $\begin{array}{l}\text { Serum uric acid, } \mu \mathrm{mmol} / \mathrm{l} \\
(\text { mean } \pm \mathrm{SD})\end{array}$ & $352.6 \pm 121.5$ & $380.8 \pm 106.9$ & 0.005 & $336.6 \pm 109.1$ & $383.0 \pm 118.1$ & 0.013 \\
\hline $\mathrm{PT}, \sec ($ mean $\pm \mathrm{SD})$ & $13.7 \pm 4.6$ & $13.2 \pm 3.0$ & 0.367 & $13.6 \pm 1.8$ & $14.4 \pm 1.7$ & 0.164 \\
\hline APTT, sec $($ mean \pm SD) & $38.3 \pm 10.4$ & $39.4 \pm 21.3$ & 0.741 & $45.8 \pm 27.5$ & $47.9 \pm 24.9$ & 0.830 \\
\hline $\mathrm{CK}-\mathrm{Mb}, \mathrm{u} / \mathrm{l}(\mathrm{mean} \pm \mathrm{SD})$ & $19.6 \pm 34.8$ & $27.1 \pm 91.3$ & 0.280 & $91.0 \pm 124.5$ & $82.4 \pm 158.2$ & 0.725 \\
\hline TnT, pg/ml (median, IQR) & $15.8(8.9-49.1)$ & $16.4(10.8-66.4)$ & 0.380 & $\begin{array}{c}3092.5 \\
(2437.8-4858.0)\end{array}$ & $\begin{array}{c}2117.0 \\
(1057.9-4761.0)\end{array}$ & 0.288 \\
\hline hs-CRP, mg/l (median, IQR) & $2.7(1.1-15.1)$ & $3.4(1.1-12.9)$ & 0.813 & $24.5(12.1-44.8)$ & $14.9(4.4-55.7)$ & 0.328 \\
\hline $\begin{array}{l}\text { NT-proBNP, pg/ml (median, } \\
\text { IQR) }\end{array}$ & $\begin{array}{c}644.5 \\
(225.5-2074.6)\end{array}$ & $\begin{array}{c}755.3 \\
(256.7-2273.2)\end{array}$ & 0.458 & $\begin{array}{c}2751.7 \\
(1418.2-6079.7)\end{array}$ & $\begin{array}{c}3387.9 \\
(1561.7-7932.8)\end{array}$ & 0.268 \\
\hline $\mathrm{EF}, \%($ mean $\pm \mathrm{SD})$ & $55.3 \pm 10.1$ & $55.1 \pm 10.1$ & 0.862 & $47.9 \pm 9.6$ & $48.0 \pm 11.5$ & 0.961 \\
\hline \multicolumn{7}{|l|}{ In-hospital management } \\
\hline Aspirin, no. (\%) & $174(80.2)$ & $261(83.9)$ & 0.267 & $53(94.6)$ & $115(92.0)$ & 0.524 \\
\hline Clopidogrel, no. (\%) & $183(84.7)$ & $273(87.8)$ & 0.312 & $51(91.1)$ & $118(93.7)$ & 0.533 \\
\hline ACEI/ARB, no. (\%) & $129(59.4)$ & $190(60.9)$ & 0.737 & $39(69.6)$ & $78(61.9)$ & 0.315 \\
\hline Beta blocker, no. (\%) & $167(77.0)$ & $218(69.9)$ & 0.072 & $39(69.6)$ & $88(69.8)$ & 0.979 \\
\hline Statin, no. (\%) & $210(97.7)$ & $298(95.5)$ & 0.191 & $55(98.2)$ & $124(98.4)$ & 0.923 \\
\hline Diuretic, no. (\%) & $93(43.7)$ & $124(40.4)$ & 0.457 & $35(62.5)$ & $86(68.8)$ & 0.405 \\
\hline PPI, no. (\%) & $173(81.2)$ & $223(72.6)$ & 0.024 & $54(96.4)$ & $117(93.6)$ & 0.441 \\
\hline IABP, no. (\%) & $1(0.5)$ & $8(2.6)$ & 0.089 & $6(10.7)$ & $10(8.3)$ & 0.609 \\
\hline Revascularization & $49(22.6)$ & $114(36.5)$ & 0.002 & $33(41.1)$ & $60(47.6)$ & 0.159 \\
\hline In-hospital events & & & & & & \\
\hline
\end{tabular}


TABle 2: Continued.

\begin{tabular}{|c|c|c|c|c|c|c|}
\hline & \multicolumn{3}{|c|}{ NSTE-ACS } & \multicolumn{3}{|c|}{ STEMI } \\
\hline & Women $(n=217)$ & Men $(n=312)$ & $p$ & Women $(n=56)$ & Men $(n=126)$ & $p$ \\
\hline Heart failure, no. (\%) & $25(11.8)$ & $29(9.5)$ & 0.404 & $17(30.9)$ & $48(38.4)$ & 0.335 \\
\hline Bleeding, no. (\%) & $14(6.6)$ & $17(5.6)$ & 0.618 & $8(14.3)$ & $22(17.6)$ & 0.579 \\
\hline $\begin{array}{l}\text { Ventricular tachycardia, no. } \\
(\%)\end{array}$ & $16(7.5)$ & $19(6.2)$ & 0.554 & $7(12.5)$ & $17(13.6)$ & 0.840 \\
\hline Stroke, no. (\%) & $3(1.4)$ & $1(0.3)$ & 0.310 & $2(3.6)$ & $1(0.8)$ & 0.177 \\
\hline Death, no. (\%) & $2(2.2)$ & $4(3.0)$ & 0.719 & $2(8.7)$ & $4(8.7)$ & 1.000 \\
\hline \multicolumn{7}{|l|}{ One-year follow-up } \\
\hline Revascularization & $4(2.0)$ & $6(2.2)$ & 0.891 & $0(0.0)$ & $1(1.1)$ & 0.480 \\
\hline Readmission, no. (\%) & $78(37.3)$ & $119(40.5)$ & 0.475 & $18(35.3)$ & $40(38.5)$ & 0.702 \\
\hline Stroke, no. (\%) & $10(4.9)$ & $16(5.7)$ & 0.698 & $3(6.4)$ & $2(2.1)$ & 0.193 \\
\hline Death, no. (\%) & $29(13.4)$ & $47(15.1)$ & 0.583 & $11(19.6)$ & $48(38.1)$ & 0.014 \\
\hline
\end{tabular}

LDL-C $(2.4 \pm 0.9$ vs. $2.2 \pm 0.8, p=0.002)$, and HDL-C $(1.1 \pm 0.3$ vs. $1.0 \pm 0.3, p=0.002)$ were significantly higher, whereas hemoglobin levels $(110.4 \pm 18.3$ vs. $121.8 \pm 20.2$, $p<0.001)$, creatinine levels $(89.9 \pm 54.1$ vs. $124.4 \pm 98.6$, $p<0.001)$, and serum uric acid (349.2 \pm 119.1 vs. $381.4 \pm 110.1, p<0.001)$ were significantly lower in women than in men. Regarding in-hospital management, the coronary revascularization rate was significantly lower in women than in men $(30.0 \%$ vs. $39.7 \%, p=0.022)$, while the medication rate and rate of intra-aortic balloon pump (IABP) use were similar between the two groups. There were no differences in in-hospital adverse events. A total of 135 patients died (19.0\%), and the 1-year mortality rate was significantly lower in women than in men (14.7\% vs. $21.7 \%$, $p=0.020)$. The percentages of rehospitalization, revascularization, stroke, and bleeding after discharge were similar between the two groups.

3.3. Differences between Women and Men according to ACS Type. The results of the subgroup (NSTE-ACS and STEMI) comparisons are shown in Table 2. There were 182 (25.8\%) patients with STEMI and 529 (74.2\%) with NSTEACS. Among the 256 (36\%) patients who underwent coronary revascularization, 93 (51.1\%) were STEMI patients, and 163 (30.8\%) were NSTE-ACS patients. Sexrelated differences, including the prevalence of current smoking, and hemoglobin, platelet, total cholesterol, LDL$\mathrm{C}$, creatinine, and serum uric acid levels were still observed in both STEMI and NSTE-ACS subgroups. In the NSTEACS subgroup, the rates of hyperuricemia (41.9\% vs. $31.4 \%, p=0.013)$, severe heart dysfunction on admission $(60.8 \%$ vs. $47.9 \%, p=0.003)$, high HDL-C ( $1.1 \pm 0.3$ vs. $1.0 \pm 0.3, p=0.002)$, and the use of proton pump inhibitors (PPIs) (81.2\% vs. $72.6 \%, p=0.024)$ were significantly higher, while the coronary revascularization rate $(22.6 \%$ vs. $36.5 \%, p=0.002$ ) was lower in women than in men, but these differences were not observed in the STEMI subgroup. In patients with STEMI, the rate of diabetes mellitus $(48.2 \%$ vs. $19.8 \%, p<0.001)$ was higher in women than in men and 1-year mortality $(19.6 \%$ vs. $38.1 \%$, $p=0.014$ ) was lower.
3.4. Multivariable Analyses between Women and Men. To evaluate whether the residual sex difference in mortality could be explained by disparities in the risk factors, we examined the independent determinants of 1-year all-cause mortality. As shown in Table 3, female patients had a significantly lower unadjusted risk of death (unadjusted $\mathrm{OR}=0.620,95 \%$ CI $0.413-0.929, p=0.021$ ) than male patients. The multivariable logistic regression analysis showed similar results $(\mathrm{OR}=0.597,95 \%$ CI $0.397-0.900, p=0.014)$ after adjusting for age (Model 1). Additional variables included diabetes mellitus, hypertension, current smoking, and severe heart failure (Model 2) and hemoglobin, platelet, total cholesterol, HDL-C, LDL-C, creatinine, and serum uric acid levels (Model 3). The same associations were observed in Model $2(\mathrm{OR}=0.531,95 \% \mathrm{CI} 0.347-0.811, p=0.003)$ and Model 3 (OR $=0.565,95 \%$ CI 0.351-0.908, $p=0.018)$. Similar results were observed in elderly patients with STEMI but not in elderly patients with NSTE-ACS.

Regarding the adjustment variables in Model 3, in all patients, age $(\mathrm{OR}=1.08,95 \% \mathrm{CI}: 1.02-1.15, p=0.010)$, severe heart failure $(\mathrm{OR}=1.77,95 \% \mathrm{CI}: 1.14-2.75, p=0.011)$, PLT levels (OR $=1.004,95 \% \mathrm{CI}: 1.001-1.007, p=0.018)$, and creatinine levels $(\mathrm{OR}=1.005,95 \% \quad \mathrm{CI}$ : 1.003-1.007, $p<0.001)$ were associated with mortality, along with sex (OR $=0.57,95 \% \mathrm{CI}: 0.35-0.91, p=0.018$ ). In patients with STEMI, severe heart failure $(\mathrm{OR}=3.84,95 \% \mathrm{CI}$ : $1.89-7.78$, $p<0.001)$ was associated with mortality, along with sex (OR $=0.42,95 \% \mathrm{CI}: 0.18-0.94, p=0.035)$. In patients with NSTE-ACS, age (OR=1.09, 95\% CI: $1.01-1.18, p=0.033)$, hemoglobin $(\mathrm{OR}=0.98,95 \% \mathrm{CI}: 0.97-1.00, p=0.027)$, LDL (OR $=1.49,95 \% \mathrm{CI}: 1.07-2.08, p=0.018)$, and creatinine (OR $=1.004,95 \%$ CI: $1.002-1.007, p=0.001)$ were associated with mortality.

\section{Discussion}

The main findings of the present study are as follows: (1) elderly women had more comorbidities and were more likely to present with NSTE-ACS than men in the total ACS cohort; (2) in women with NSTE-ACS, the prevalence of severe heart failure on admission was higher, and they underwent coronary revascularization less often than men, 
TABLE 3: Multivariable logistic regression analysis of one-year all-cause mortality.

\begin{tabular}{|c|c|c|c|c|c|c|c|c|c|c|c|c|}
\hline & \multicolumn{3}{|c|}{ Unadjusted } & \multicolumn{3}{|c|}{ Model 1} & \multicolumn{3}{|c|}{ Model 2} & \multicolumn{3}{|c|}{ Model 3} \\
\hline & OR & $95 \% \mathrm{CI}$ & $p$ & OR & $95 \% \mathrm{CI}$ & $p$ & OR & $95 \% \mathrm{CI}$ & $p$ & OR & $95 \% \mathrm{CI}$ & $p$ \\
\hline \multicolumn{13}{|l|}{ Total } \\
\hline Women vs. men & 0.62 & $0.41-0.93$ & 0.021 & 0.60 & $0.40-0.90$ & 0.014 & 0.53 & $0.35-0.81$ & 0.003 & 0.57 & $0.35-0.91$ & 0.018 \\
\hline Age & - & - & - & 1.10 & $1.04-1.16$ & 0.001 & 1.09 & $1.03-1.15$ & 0.003 & 1.08 & $1.02-1.15$ & 0.010 \\
\hline Severe heart failure & - & - & - & - & - & - & - & - & - & 1.77 & $1.14-2.75$ & 0.011 \\
\hline PLT & - & - & - & - & - & - & - & - & - & 1.004 & $1.001-1.007$ & 0.018 \\
\hline Creatinine & - & - & - & - & - & - & - & - & - & 1.005 & $1.003-1.007$ & $<0.001$ \\
\hline \multicolumn{13}{|l|}{ STEMI } \\
\hline Women vs. men & 0.40 & $0.19-0.84$ & 0.016 & 0.40 & $0.19-0.84$ & 0.016 & 0.36 & $0.17-0.79$ & 0.011 & 0.42 & $0.18-0.94$ & 0.035 \\
\hline Severe heart failure & - & - & - & - & - & - & 3.21 & $1.66-6.22$ & 0.001 & 3.84 & $1.89-7.78$ & $<0.001$ \\
\hline \multicolumn{13}{|l|}{ NSTE-ACS } \\
\hline Women vs. men & 0.87 & $0.53-1.43$ & 0.584 & 0.81 & $0.49-1.34$ & 0.405 & 0.72 & $0.43-1.23$ & 0.230 & 0.71 & $0.38-1.33$ & 0.283 \\
\hline Age & - & - & - & 1.13 & $1.05-1.20$ & $<0.001$ & 1.10 & $1.02-1.18$ & 0.009 & 1.09 & $1.01-1.18$ & 0.033 \\
\hline Severe heart failure & - & - & - & - & - & - & 1.79 & $1.05-3.07$ & 0.033 & - & - & - \\
\hline Hemoglobin & - & - & - & - & - & - & - & - & - & 0.98 & $0.97-1.00$ & 0.027 \\
\hline LDL & - & - & - & - & - & - & - & - & - & 1.49 & $1.07-2.08$ & 0.018 \\
\hline Creatinine & - & - & - & - & - & - & - & - & - & 1.004 & $1.002-1.007$ & 0.001 \\
\hline Serum uric acid & - & - & - & - & - & - & - & - & - & 1.002 & $1.000-1.004$ & 0.090 \\
\hline
\end{tabular}

Data are expressed as OR $\pm 95 \%$ CIs (reported in parentheses) as assessed by univariate (unadjusted) or multivariate logistic regression analyses. Other covariates included in multivariate logistic regression models were as follows: Model 1: age; Model 2: age, diabetes mellitus, hypertension, current smoking, and severe heart failure; Model 3: adjustment for variables included age, diabetes mellitus, hypertension, current smoking, severe heart failure, hemoglobin, PLT, total cholesterol, HDL-C, LDL-C, creatinine level, and serum uric acid. The adjustment parameters which were statistically significant were shown.

but in-hospital adverse events and 1-year mortality were similar between women and men; (3) in STEMI patients, there were no differences in the in-hospital treatments and in-hospital adverse events between women and men, but women had lower 1-year mortality than men; and (4) female sex was a protective factor for 1-year mortality in all populations, especially in patients with STEMI.

Although traditional atherosclerotic disease risk factors are important for both men and women with ACS, some factors accumulated more often in female patients. In the present study, women had higher rates of traditional risk factors, including hypertension, diabetes, hyperuricemia, elevated LDL-C, and severe heart failure, than men. In contrast, men were more likely to be smokers and have elevated creatinine levels. Notably, these differences were similar across both types of ACS (STEMI and NSTE-ACS). These risk factor distribution patterns have been confirmed by other studies in the whole populations [7] and in elderly patients $[4,9]$. In addition, women have sex-specific risk factors, such as pregnancy and menopause $[7,13]$. It is accepted that the cardiovascular risk profile of women worsens during postmenopause, and the prevalence of coronary artery disease (CAD) steeply increases with age thereafter. We also observed that among the elderly patients with ACS, women were more likely to present with NSTEACS than men, whereas men presented with STEMI more often than women. This finding is in agreement with data derived from the Improving Care for Cardiovascular Disease in China (CCC) Project, in that the prevalence of STEMI was significantly lower in women than in men, and women were more likely to present with NSTE-ACS compared to their male counterparts [4]. An increased burden of plaque erosion, coronary vasospasm, spontaneous coronary artery dissection, and stress-related cardiomyopathy in women might partly be related to the observed phenomenon [7].
In terms of in-hospital management among patients with NSTE-ACS, there were no differences between women and men, except for the use of PPIs. We believe that the use of PPIs occurred more often in women than in men, which could be explained by the increased bleeding risk in women with ACS $[8,14,15]$. Nevertheless, no difference was observed regarding in-hospital bleeding in the present study; the use of PPIs might partly contribute to this finding. In the present study, we observed a lower coronary revascularization rate in women hospitalized for NSTE-ACS than in men. This result is in line with data from the CCC project, which showed that eligible women with NSTE-ACS were less likely to undergo timely percutaneous coronary intervention (PCI) than men with NSTE-ACS (30.5\% vs. $34.2 \%, p<0.001$ ) [4]. We believe that the fear of complications associated with invasive treatments might, in part, explain this finding because women, especially older women, might be considered too fragile to undergo aggressive treatments. A surprising finding in our study was the similar in-hospital clinical outcomes between women and men, despite fewer PCI carried out in women. This finding was in contrast with a previous report that showed that women with NSTE-ACS had higher crude in-hospital mortality rates than men with NSTE-ACS [4]. Because our cohort included only patients of $\geq 75$ years of age, the above difference might originate from the different age groups. In addition, a recent report confirmed that women with NSTE-ACS $\geq 70$ years of age had better outcomes than those $<70$ years [8]. Another study using data from the National Inpatient Sample (NIS) database in the United States indicated that women had lower risk-adjusted in-hospital mortality than men after accounting for differences in age and comorbidities [16]. These findings suggested that the relation between sex and mortality was age-dependent, with increased mortality in women at a young age and decreased mortality in women at an advanced age. 
Among the patients with STEMI, there were no differences regarding in-hospital treatments and in-hospital adverse events between women and men, but women had better 1-year outcomes than men in the present study. Some studies demonstrated increased rates of mortality among women, some studies indicated no difference, and other studies showed lower rates of mortality in women than in men [17]. These controversial results may be explained by potential interactions between age and sex; significant differences in in-hospital mortality rates between women and men with STEMI were demonstrated when the cohort was stratified by age groups ( $<55$ years, 55-64 years, and $>75$ years) [4]. Younger age was associated with higher 30-day mortality rates in women with STEMI, but this difference decreased after age 60 and was no longer observed in elderly women [18]. In fact, mortality in elderly women was lower than that in age-matched men, as shown by previous studies $[19,20]$; this result was further confirmed by the Netherlands National Trial Register, which showed that excess mortality in women mostly occurred in young patients with STEMI, while older women had a better outcome than men of the same age [9]. These findings suggest that there is an age-dependent relationship among the outcomes between male and female patients with STEMI. Our results show that elderly female patients with STEMI have lower 1-year mortality than elderly male patients with STEMI.

Previous studies have shown that sex differences in early mortality after ACS could be largely explained by the clinical differences at presentation $[21,22]$. To evaluate whether the sex differences associated with 1-year mortality could be explained by disparities in clinical characteristics, we adjusted for comorbidities, presentation, and clinical profiles. After adjusting for age (Model 1); age, diabetes mellitus, hypertension, current smoking, and severe heart failure (Model 2); and hemoglobin, platelet counts, total cholesterol, HDL-C, LDL-C, creatinine, and serum uric acid (Model 3), the female sex was consistently shown as an independent protective factor for 1-year mortality in the whole cohort, especially among patients with STEMI. Nevertheless, a delay in presentation $[1,4,23]$ and angiographic severity of coronary lesions [17], which may also contribute to the sex difference in mortality after ACS, were not adjusted in the present study. In addition, a previous study indicated that the more favorable mortality rate in older women could be attributed to the shorter exposure to obstructive coronary disease and longer life expectancy in women than in men [18]. In all patients, sex, age, severe heart failure, PLT, and creatinine were all independently associated with mortality. In patients with STEMI, only sex and severe heart failure were independently associated with mortality. Since all patients were menopausal, the differences cannot be attributed to estrogens, and other factors are also involved in the mortality risk. In patients with NSTE-ACS, age, hemoglobin, LDL, and creatinine were independently associated with mortality, but not sex. Therefore, in NSTEACS, other traditional risk factors for mortality play more important roles in the risk of mortality. Nevertheless, the mechanism for the sex disparity in 1-year mortality, especially in elderly patients with STEMI, still needs to be further investigated in future studies.

\section{Limitations}

This study has some limitations. First, this was a singlecenter experience and included a small number of patients. Second, residual measured and unmeasured confounding factors, including changes in ECG parameters, might have contributed to some of these findings but were not included in the regression model. Third, the data were based on the routine clinical parameters measured in the management of ACS, which do not include sexual hormone levels. Finally, the details of the procedural characteristics, especially the time intervals for STEMI and angiographic severity of coronary lesions, are important in view of the previously described sex differences in the literature, but these factors were not analyzed in this study.

\section{Conclusion}

Our study showed that elderly women with ACS had more comorbidities and were more likely to present with NSTEACS than men in the total cohort, similar to other studies. A surprising finding was the better 1-year outcome in elderly women with STEMI than in elderly men with STEMI, while in-hospital and 1-year outcomes were similar between elderly women and men with NSTE-ACS. It is worth noting that the female sex was an independent protective factor for 1-year mortality in the whole ACS cohort, especially in patients with STEMI.

\section{Data Availability}

The data used to support the findings of this study are included within the Supplementary Materials.

\section{Conflicts of Interest}

The authors declare that they have no conflicts of interest regarding the publication of this paper.

\section{Acknowledgments}

This research was supported by the Natural Science Foundation of China (Grants nos. 81670269 to Shenghua Zhou and 81801394 to Shi Tai).

\section{Supplementary Materials}

A supplementary Excel datasheet file was used for statistical analysis. (Supplementary Materials)

\section{References}

[1] E. J. Benjamin, S. S. Virani, C. W. Callaway et al., "Heart disease and stroke statistics-2018 update: a report from the American heart association," Circulation, vol. 137, no. 12, pp. e67-e492, 2018. 
[2] X. Dai, J. Busby-Whitehead, and K. P. Alexander, "Acute coronary syndrome in the older adults," Journal of Geriatric Cardiology: JGC, vol. 13, no. 2, pp. 101-108, 2016.

[3] D. D. McManus, J. Gore, J. Yarzebski, F. Spencer, D. Lessard, and R. J. Goldberg, "Recent trends in the incidence, treatment, and outcomes of patients with STEMI and NSTEMI," The American Journal of Medicine, vol. 124, no. 1, pp. 40-47, 2011.

[4] Y. Hao, J. Liu, J. Liu et al., "Sex differences in in-hospital management and outcomes of patients with acute coronary syndrome," Circulation, vol. 139, no. 15, pp. 1776-1785, 2019.

[5] L. Mandelzweig, A. Battler, V. Boyko et al., "The second Euro heart survey on acute coronary syndromes: characteristics, treatment, and outcome of patients with ACS in Europe and the mediterranean basin in 2004," European Heart Journal, vol. 27, no. 19, pp. 2285-2293, 2006.

[6] M. Liakos and P. B. Parikh, "Gender disparities in presentation, management, and outcomes of acute myocardial infarction," Current Cardiology Reports, vol. 20, no. 8, p. 64, 2018.

[7] N. J. Pagidipati and E. D. Peterson, "Acute coronary syndromes in women and men," Nature Reviews Cardiology, vol. 13, no. 8, pp. 471-480, 2016.

[8] M. De Carlo, N. Morici, S. Savonitto et al., "Sex-related outcomes in elderly patients presenting with non-ST-segment elevation acute coronary syndrome: insights from the Italian elderly ACS study," JACC: Cardiovascular Interventions, vol. 8, no. 6, pp. 791-796, 2015.

[9] M. E. T. Haaf, M. Bax, J. M. T. Berg et al., "Sex differences in characteristics and outcome in acute coronary syndrome patients in The Netherlands," Netherlands Heart Journal, vol. 27, no. 5, pp. 263-271, 2019.

[10] P. T. O'Gara, F. G. Kushner, D. D. Ascheim et al., “2013 ACCF/AHA guideline for the management of ST-elevation myocardial infarction: a report of the American college of cardiology foundation/American heart association task force on practice guidelines," Circulation, vol. 127, no. 4, pp. e362-425, 2013.

[11] E. A. Amsterdam, N. K. Wenger, R. G. Brindis et al., "2014 AHA/ACC guideline for the management of patients with non-ST-elevation acute coronary syndromes: a report of the American college of cardiology/American heart association task force on practice guidelines," Journal of the American College of Cardiology, vol. 64, no. 24, pp. e139-e228, 2014.

[12] R. Mehran, S. V. Rao, D. L. Bhatt et al., "Standardized bleeding definitions for cardiovascular clinical trials: a consensus report from the bleeding academic research consortium," Circulation, vol. 123, no. 23, pp. 2736-2747, 2011.

[13] H. Wada, K. Miyauchi, and H. Daida, "Gender differences in the clinical features and outcomes of patients with coronary artery disease," Expert Review of Cardiovascular Therapy, vol. 17, no. 2, pp. 127-133, 2019.

[14] P. Kaul, J.-F. Tanguay, L. K. Newby et al., “Association between bleeding and mortality among women and men with high-risk acute coronary syndromes: insights from the early versus delayed, provisional eptifibatide in acute coronary syndromes (EARLY ACS) trial," American Heart Journal, vol. 166, no. 4, pp. 723-728, 2013.

[15] B. Ahmed, W. D. Piper, D. Malenka et al., "Significantly improved vascular complications among women undergoing percutaneous coronary intervention: a report from the Northern New England percutaneous coronary intervention registry," Circulation: Cardiovascular Interventions, vol. 2, no. 5, pp. 423-429, 2009.
[16] T. Gupta, D. Kolte, S. Khera et al., "Contemporary sex-based differences by age in presenting characteristics, use of an early invasive strategy, and inhospital mortality in patients with non-ST-segment-elevation myocardial infarction in the United States," Circulation: Cardiovascular Interventions, vol. 11, no. 1, Article ID e005735, 2018.

[17] J. S. Berger, L. Elliott, D. Gallup et al., "Sex differences in mortality following acute coronary syndromes," Journal of the American Medical Association, vol. 302, no. 8, pp. 874-882, 2009.

[18] E. Cenko, J. Yoon, S. Kedev et al., "Sex differences in outcomes after STEMI: effect modification by treatment strategy and age," JAMA Internal Medicine, vol. 178, no. 5, pp. 632-639, 2018.

[19] M. Izadnegahdar, C. Norris, P. Kaul, L. Pilote, and K. H. Humphries, "Basis for sex-dependent outcomes in acute coronary syndrome," Canadian Journal of Cardiology, vol. 30, no. 7, pp. 713-720, 2014.

[20] A. M. Otten, A. H. Maas, J. P. Ottervanger et al., "Is the difference in outcome between men and women treated by primary percutaneous coronary intervention age dependent? Gender difference in STEMI stratified on age," European Heart Journal: Acute Cardiovascular Care, vol. 2, no. 4, pp. 334-341, 2013.

[21] U. Keil and L. Chambless, "Sex differences in mortality after myocardial infarction: is there evidence for an increased risk for women?" Circulation, vol. 92, no. 12, pp. 3576-3577, 1995.

[22] K. MacIntyre, S. Stewart, S. Capewell et al., "Gender and survival: a population-based study of 201,114 men and women following a first acute myocardial infarction," Journal of the American College of Cardiology, vol. 38, no. 3, pp. 729-735, 2001.

[23] P. P. Mohanan, R. Mathew, S. Harikrishnan et al., "Presentation, management, and outcomes of 25748 acute coronary syndrome admissions in Kerala, India: results from the Kerala ACS registry," European Heart Journal, vol. 34, no. 2, pp. 121-129, 2013. 